\title{
X-Ray-Sensitive Hybrid Photon Detectors With Be-Windows
}

\author{
Carmelo D'Ambrosio, F. De Notaristefani, H. Leutz, D. Puertolas, and E. Rosso
}

\begin{abstract}
A 0.3-mm thin $\mathrm{YAlO}_{3}(\mathrm{Ce})$ scintillating crystal plate of $22 \mathrm{~mm}$ diameter was mounted inside an electrostatic-focussed hybrid photomultiplier tube (HPMT). The photocathode was evaporated directly on this scintillating plate opposite to the HPMT-anode. The HPMT was vacuum-sealed with a 1-mm thick Be radiation entrance window. Photoelectron numbers at their peak energies of photoabsorption and energy resolutions were measured between 3.69 and $59.6 \mathrm{keV}$. The average number of photoelectrons produced per $\mathrm{keV}$ radiation energy was determined to be $4.457 \mathrm{phel} / \mathrm{keV}$ and the energy resolution at $59.6 \mathrm{keV}$ was $17 \%$. Introducing in the same way such a thin $\mathrm{YAlO}_{3}(\mathrm{Ce})$ plate inside an imaging silicon pixel array (ISPA) tube would improve its spatial resolution, since it depends on the thickness of the $\mathrm{X}$-ray detector.
\end{abstract}

Index Terms-29.30 spectroscopic techniques, 29.40 radiation detectors, 85.60.H photodetectors, hybrid photon detectors, YAP(Ce).

\section{INTRODUCTION}

$\mathbf{F}$ OR X-ray radiography we developed a new version of hybrid photon detector (HPD). The light transparent entrance window, which vacuum-seal this tube, is replaced by a thin Be-foil. It transmits low energy X-rays to a thin scintillating $\mathrm{YAP}(\mathrm{Ce})$ plate $^{1}[1]$, mounted inside the tube, which, if used as an entrance window, would not resist the atmospheric pressure. The photocathode is directly evaporated on the YAP surface opposing the HPD anode.

Thin YAP $(\mathrm{Ce})$ plates offer two advantages for low energy radiation detection: 1) They cause less self-absorption of their scintillation and 2) improve the spatial resolution, as both depend on their thickness [1]. This paper reports on the first application of this new HPD concept. We tested it with an electrostatically-focussed hybrid photomultiplier tube (HPMT) [1], an HPD version of comparatively simple design, and therefore, appropriate for the first test. To verify the first advantage, we measured photoelectron numbers and energy resolutions between $3.7 \mathrm{keV}\left(\mathrm{Ca} \mathrm{K}_{\alpha}\right)$ and $59.6 \mathrm{keV}\left({ }^{241} \mathrm{Am}\right)$. The results are compared with those obtained by M. Moszynski et al. [2] and M. Kapusta et al. [3], who applied a $10 \times 10 \mathrm{~mm}^{2}$ YAP-crystal of $5 \mathrm{~mm}$ and a $3 \times 20 \mathrm{~mm}^{2}$ YAP-crystal of $3 \mathrm{~mm}$ thickness, respectively. In both cases the YAP crystals were coupled to a XP2020Q photomultiplier.

Manuscript received September 14, 2004; revised November 19, 2004 and February 3, 2005

C. D'Ambrosio, H. Leutz, and E. Rosso are with CERN, CH-1211, Geneva 23, Switzerland (e-mail: carmelo.d'ambrosio@cern.ch).

F. De Notaristefani and D. Puertolas are with INFN, Sezione di Roma, Rome, Italy.

Digital Object Identifier 10.1109/TNS.2005.850489

${ }^{1}$ Cerium doped $\mathrm{YAlO}_{3}$-crystal, manufactured by Crytur, Turnov, Czech Rep.

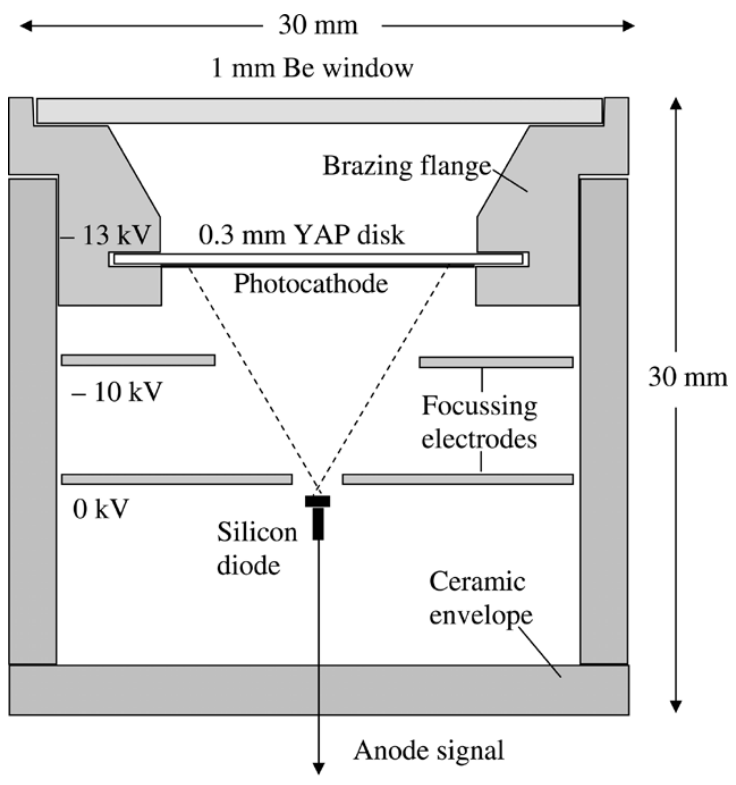

Not to scale

Fig. 1. Layout of the HPMT sealed with a 1-mm thick Be entrance window followed inside by a $0.3-\mathrm{mm}$ thick $\mathrm{YAP}(\mathrm{Ce})$ radiation detector of $22 \mathrm{~mm}$ diameter. The photocathode $(\sim 17 \mathrm{~mm}$ diameter) is directly evaporated on its surface pointing to the silicon anode ( $2 \mathrm{~mm}$ diameter) of the HPMT.

The improvement of spatial resolution (2) means an advantage for imaging silicon pixel array (ISPA) tubes [1] used for X-ray imaging. These optoelectronic cameras detect their photoelectrons with pixelized silicon anodes. The Be-window version of ISPA tubes will improve low-energy $\mathrm{X}$-ray radiography due to the thin $\mathrm{YAP}(\mathrm{Ce})$ plate. This advantage becomes increasingly important for medical or material diagnostics.

\section{Detector Layout}

The X-ray (low gamma energy) detector ${ }^{2}$ is sketched in Fig. 1. The Be-window (1-mm thick), which resists the atmospheric pressure and seals the HPMT tube, transmits electromagnetic radiation as plotted versus the energy in Fig. 2. A 0.3-mm thin $\mathrm{YAP}(\mathrm{Ce})$ plate of $22 \mathrm{~mm}$ diameter is mounted without any wrapping inside the HPMT behind the Be-window. The photoabsorption versus the radiation energy is also shown in Fig. 2 for different $\mathrm{YAP}(\mathrm{Ce})$ thicknesses. $\mathrm{YAP}(\mathrm{Ce})$ scintillates with maximum light emission at $355 \mathrm{~nm}$ and a light decay time of 27 ns. YAP is of rhombic perovskite structure, noncleavable, and hence, strongly resistant against breakage. Due to its hardness

${ }^{2}$ Manufactured by DEP, Roden, The Netherlands, with Crytur, Turnov, Czech Rep., Metaceram, Paris, France, and Kompozit, Moscow, Russian Fed. 


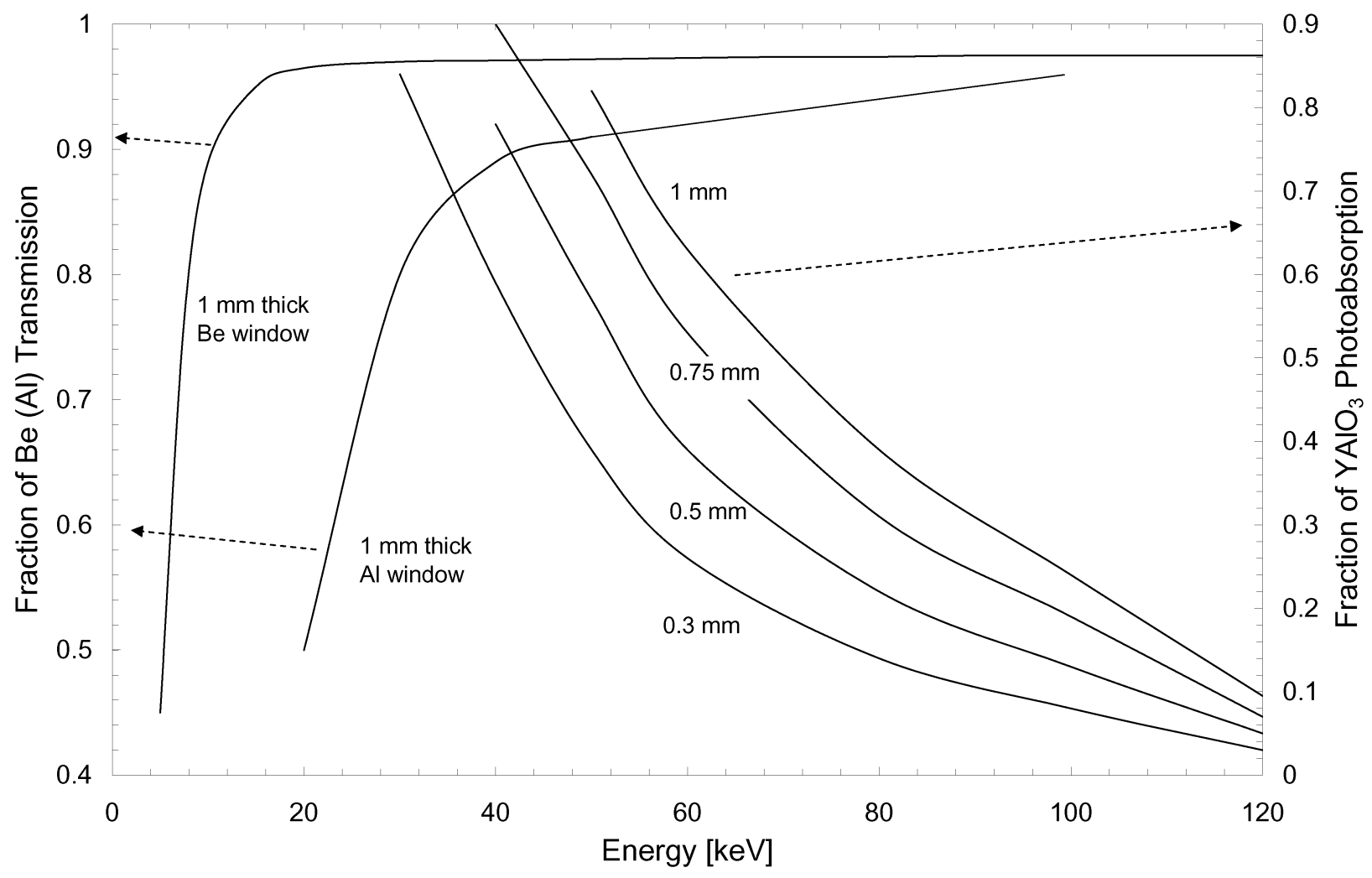

Fig. 2. Radiation transmission of the 1-mm-thick Be window (left-hand scale) and photoabsorption in YAP(Ce) plates of different thicknesses (right-hand scale). The remaining $\sim 3 \%$ in the transmission curve is due to Compton scattering processes. For comparison, the same curve for $\mathrm{Al}$ is shown.

(8.6 Mohs) it can be polished to optical grade. It is not hygroscopic and chemically inert. Since it is resistant against alkali, photocathodes can be directly evaporated on its surface [1, Table 6]. The photocathode diameter is $\sim 17 \mathrm{~mm}$.

Focusing electrodes inside the tube (Fig. 1) guide the photoelectrons with $13 \mathrm{keV}$ potential difference onto a silicon anode of $2 \mathrm{~mm}$ diameter with $1 \mathrm{pF}$ capacitance. The resulting charge signals are transmitted to a low-noise preamplifier integrated in the socket of the HPMT to avoid unnecessary cabling capacitance. The amplifier signals (shaped at $1 \mu \mathrm{s}$ ) were sent to an 8192-channels peak sensing analyzer. Photoelectron peaks were directly visualized, which allowed for a precise calibration in terms of photoelectron numbers versus the analyzer channels [4].

To verify the best potential value of the focussing electrode for achieving optimum HPMT efficiency, we varied it between $9.25 \mathrm{kV}$ and $13.75 \mathrm{kV}$ during the tube's exposure to a ${ }^{109} \mathrm{Cd}$ source $(\mathrm{Ag} \mathrm{K}, 22 \mathrm{keV})$, keeping the accelerating voltage constant at $15 \mathrm{kV}$. Two positions of the source were examined: at the center and at $\sim 7 \mathrm{~mm}$ off center of the beryllium window. Also, the ${ }^{109} \mathrm{Cd}$ source is only slightly collimated, giving an estimated spot on the $\mathrm{YAP}(\mathrm{Ce})$ crystal of $\sim 6 \mathrm{~mm}$. The results are plotted in Fig. 3(a) and show a rather weak dependence from the electrode potential. All following exposures were then performed at $10 \mathrm{kV}$ potential of the focussing electrode and $13 \mathrm{kV}$ accelerating voltage, which gives the same ratio as 11.75 and $15 \mathrm{kV}$. Fig. 3(b) displays two $\mathrm{Ag} \mathrm{K}_{\alpha}$ spectra, one taken at $11.75 \mathrm{kV}$ and the other one at $9.25 \mathrm{kV}$ potential of the focussing electrode. The difference of both distributions is obvious.

\section{Measurements AND Results}

The radioactive sources were placed according to their strengths at different distances from the Be-window of the HPMT. The characteristic $\mathrm{K}_{\alpha}$-emissions of titanium $(4.50 \mathrm{keV})$, scandium (4.09 keV) and calcium (3.69 keV) were excited by the $\mathrm{K}$-emissions of manganese following the electron capture of ${ }^{55} \mathrm{Fe}$ with $\mathrm{K}_{\alpha}=5.96 \mathrm{keV}$. Those of terbium (44.4 keV), barium $(32.0 \mathrm{keV})$, silver $(22.0 \mathrm{keV})$, molybdenum $(17.4 \mathrm{keV})$, rubidium $(13.39 \mathrm{keV})$, and copper $(8.04 \mathrm{keV})$ were excited by the gamma emission of ${ }^{241} \mathrm{Am}(59.6 \mathrm{keV})$. Samarium $\mathrm{K}_{\alpha}$ (40.0 keV), cesium $\mathrm{K}_{\alpha}(30.9 \mathrm{keV})$, and silver $\mathrm{K}_{\alpha}(22 \mathrm{keV})$ follow the ${ }^{152} \mathrm{Eu}-,{ }^{133} \mathrm{Ba}$-, and ${ }^{109} \mathrm{Cd}$ - electron capture, respectively. According to the low intensity contributions of $\mathrm{K}_{\beta}$-emissions, we associate to all $\mathrm{K}$-peaks the $\mathrm{K}_{\alpha}$-energies $\left(\alpha_{1}+\alpha_{2}\right)$. The energy spectrum of ${ }^{241} \mathrm{Am}$ is displayed in Fig. 4(a).

To further characterize and compare the response of the $\mathrm{Be}-\mathrm{YAP}(\mathrm{Ce})$ window scheme, we also measured a few spectra with a "standard" configuration [see insert in Fig. 4(b)], which consisted in placing a small $\mathrm{YAP}(\mathrm{Ce})$ disk ( $\sim$ 8-mm diameter $\times 1$-mm thin) on the 40-mm diameter quartz window of a PP0470 HPMT (for a detailed description of this HPMT, see [5]). An aluminum foil was placed on the free face of the $\operatorname{YAP}(\mathrm{Ce})$ disk, the optical contact was achieved by using a thin layer of silicon oil, and the same radioactive sources as in the Be-YAP scheme were used. A spectrum of ${ }^{109} \mathrm{Cd}$ recorded in this condition is shown in Fig. 4(b). 


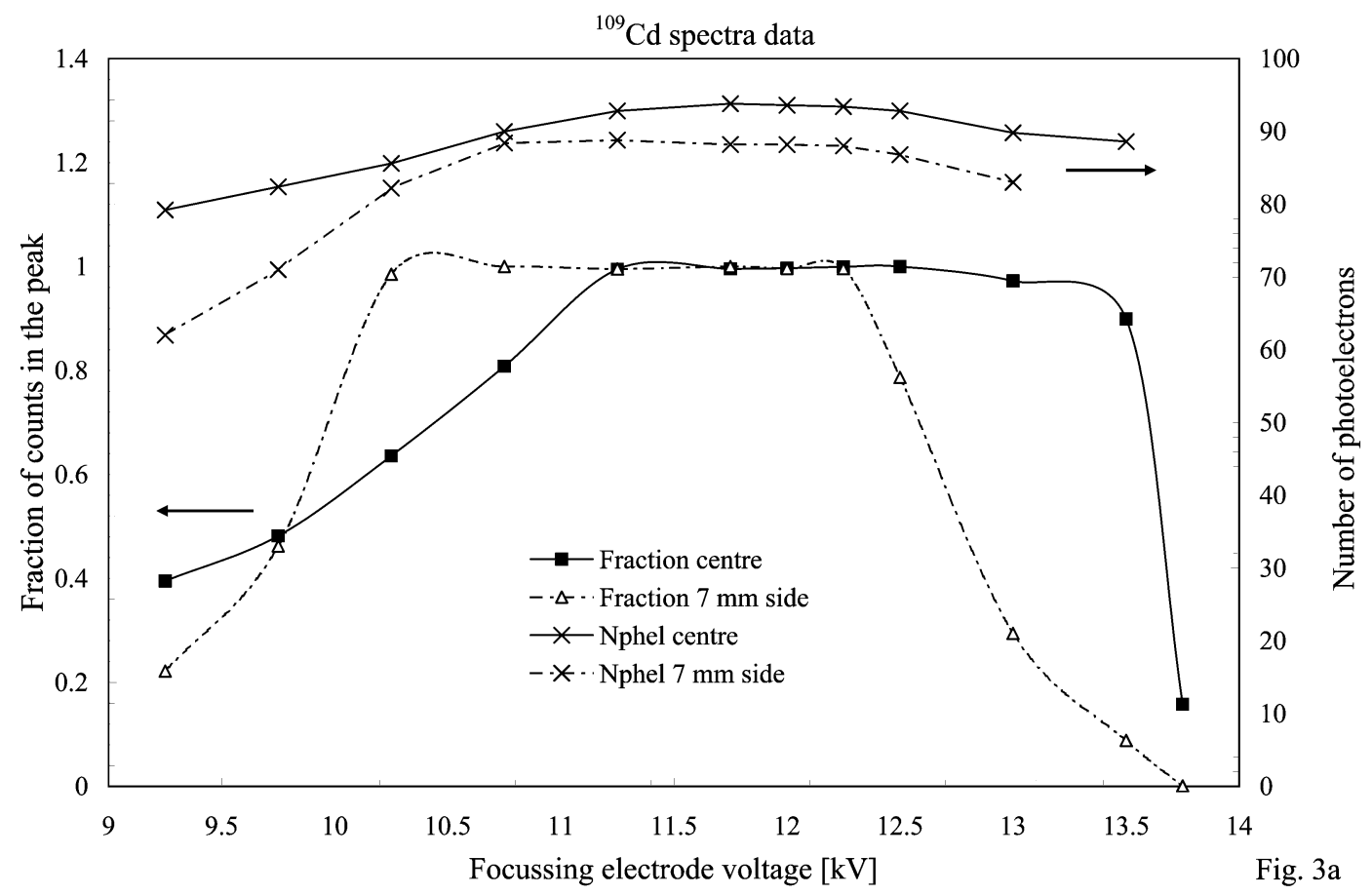

(a)

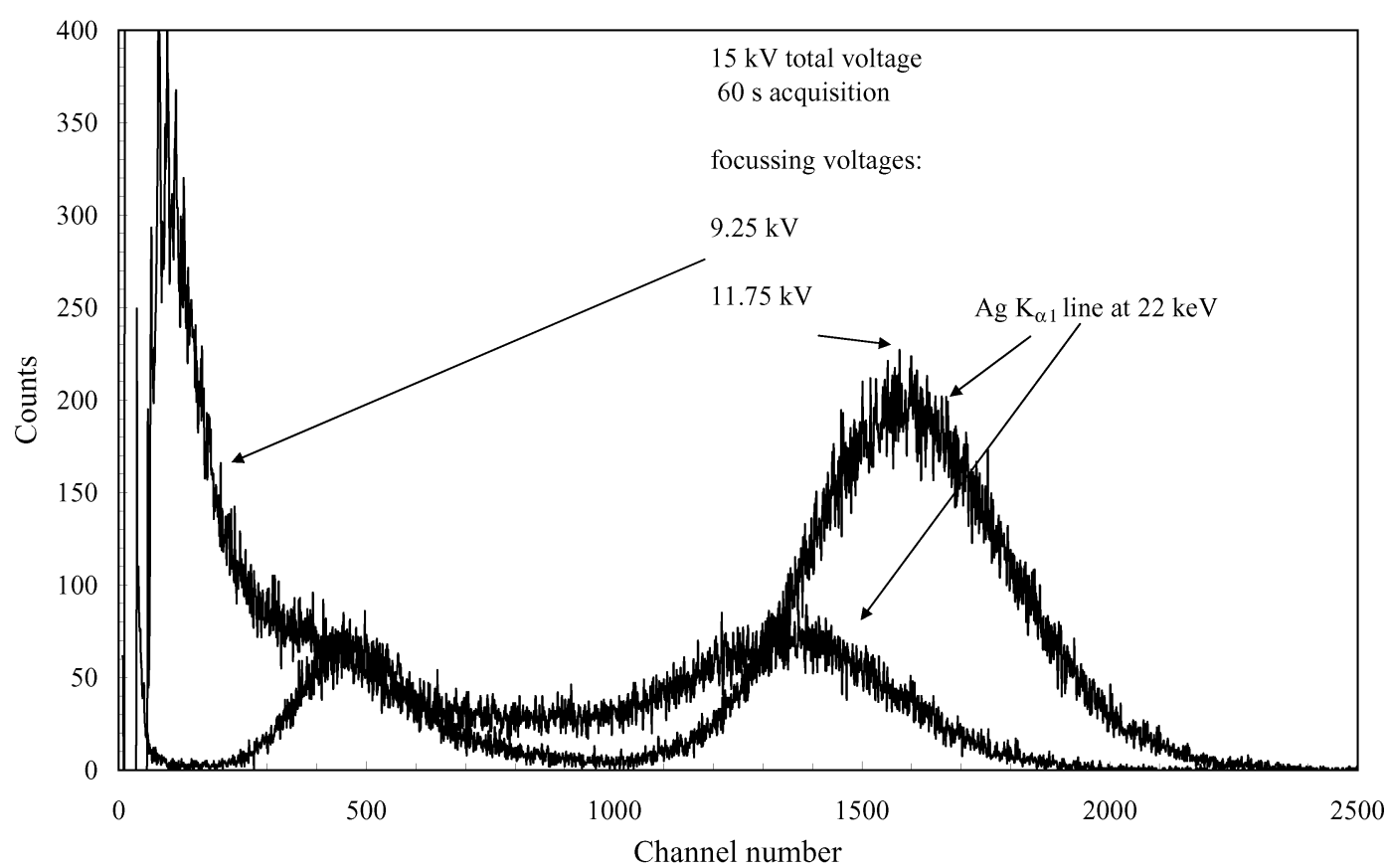

(b)

Fig. 3. (a) Dependence of number of measured photoelectrons $N_{\text {meas }}$ (right-hand scale) and counts of the total absorption peaks (left-hand scale) on the focussing potential of the Be-sealed HPMT. The ${ }^{109} \mathrm{Cd}$ source $\left(\mathrm{Ag} \mathrm{K} \mathrm{K}_{\alpha}\right)$ was placed in the center and at $\sim 7$ mm aside. Note the difference of $\sim 5$ phel in $N_{\text {meas }}$ values at the optimal voltages between center and off-center configurations. (b) Ag $\mathrm{K}_{\alpha}$ spectra at $11.75 \mathrm{kV}$ and at $9.25 \mathrm{kV}$ focussing electrode potentials are shown. Even though the average number of photoelectrons produced decreases by only $\sim 15 \%$ for $9.25 \mathrm{kV}$, the number of counts in the total absorption peak is only $40 \%$ of that measured at $11.75 \mathrm{kV}$ and the low energy background increases.

Fig. 5 (left-hand scale) shows the relation between the number of measured photoelectrons $\left(N_{\text {meas }}\right)$ and the associated peak energy of the photoabsorptions for the radiation sources mentioned above. This relation is linear through the $N_{\text {meas }}$ values. The broken line, shown in Fig. 5, is a linear regression fit to the data. It results in a slope of $4.457 \mathrm{phel} / \mathrm{keV}$ and in an intercept of $0.55 \mathrm{keV}$ (at $N_{\text {meas }}=0$ or $N_{\text {meas }}=-2.47$ phel at $E=0 \mathrm{keV}$ ). Also indicated are the measured photoelectron yield ratios (triangles, right-hand scale). Both curves show the YAP $(\mathrm{Ce})$ nonproportionality between photoelectron numbers and low-energy radiation. We observed a similar behavior with earlier measurements [4], [6]. 

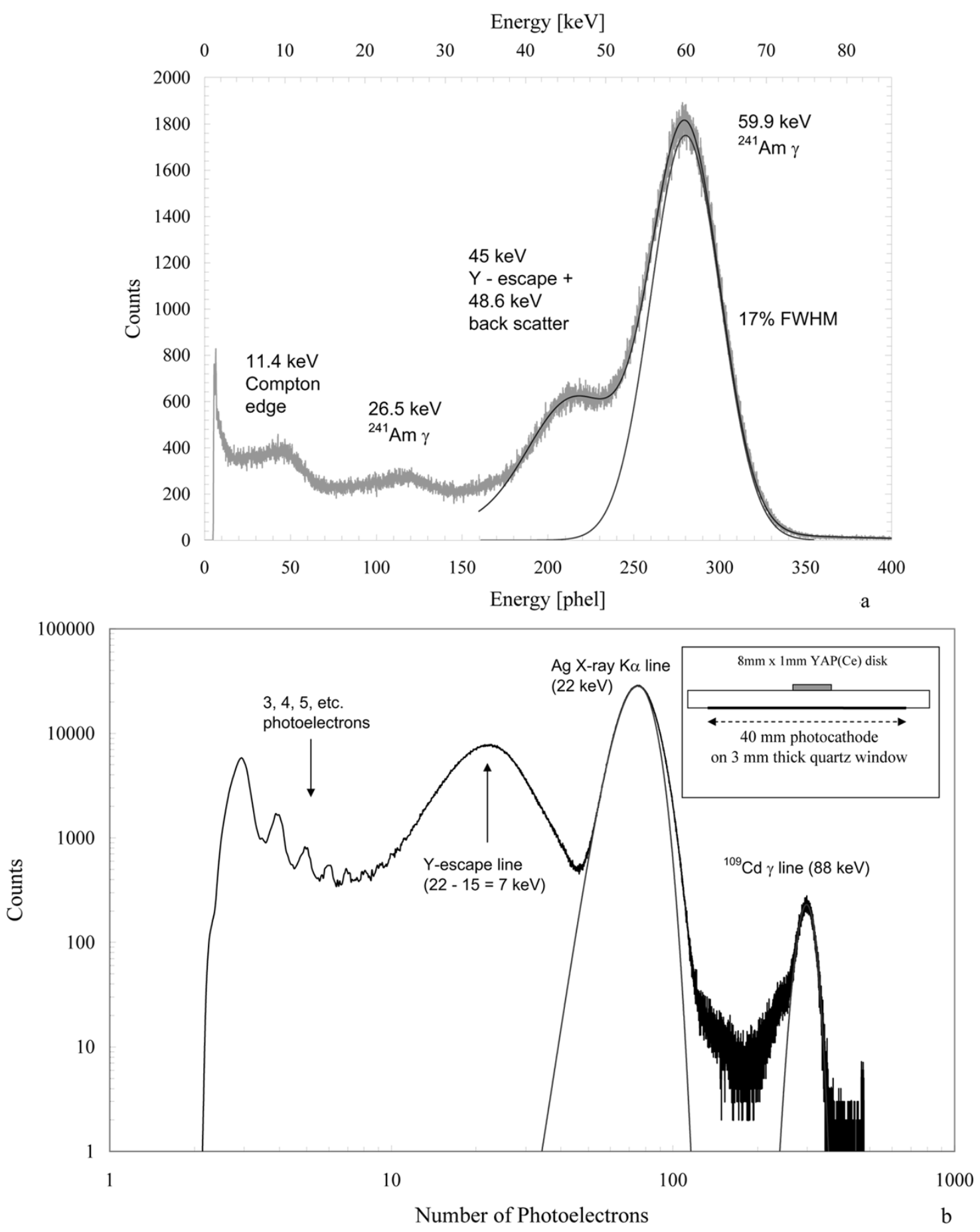

Fig. 4. (a) Spectrum of ${ }^{241} \mathrm{Am}\left(59.6 \mathrm{keV}\right.$ peak energy) measured with the Be window HPMT. b) Spectrum of ${ }^{109} \mathrm{Cd}(\mathrm{Ag} \mathrm{K} \alpha$ at $22 \mathrm{keV}$ and gamma at $88 \mathrm{keV}$ peak energies) measured with a small YAP(Ce) disk $(\sim 8 \mathrm{~mm} \times 1 \mathrm{~mm})$ placed on the quartz window of a PP0470 HPMT (see insert), called "standard" configuration in the text. Note the $3,4,5$, etc. photoelectron peaks, which allow for a precise and reliable spectrum calibration. Gaussian fits are shown for both spectra on the main peaks.

The achieved energy resolutions at FWHM (triangles) are indicated in Fig. 6 versus the photoelectron numbers (Fig. 5) associated to the X-ray energies. The same figure shows the purely statistical contribution $2.36 \times\left(N_{\text {meas }}\right)^{-1 / 2}$ (solid line) to the energy resolution. The first five triangles are extracted through a Poissonian fit of the spectrum and the rest through a Gaussian fit. All values measured are indicated in Table I.

\section{DISCUSSION}

Moszynski et al. [2] measured 4.3 photoelectrons per keV radiation energy with their 5-mm-thick $\mathrm{YAP}(\mathrm{Ce})$ plate, by comparing the position of the ${ }^{137} \mathrm{Cs}$ gamma peak $(662 \mathrm{keV})$ with that of the single photoelectron peak. Kapusta et al. [3] even measured 5.29 photoelectrons per keV averaged over $10 \gamma$ en- 


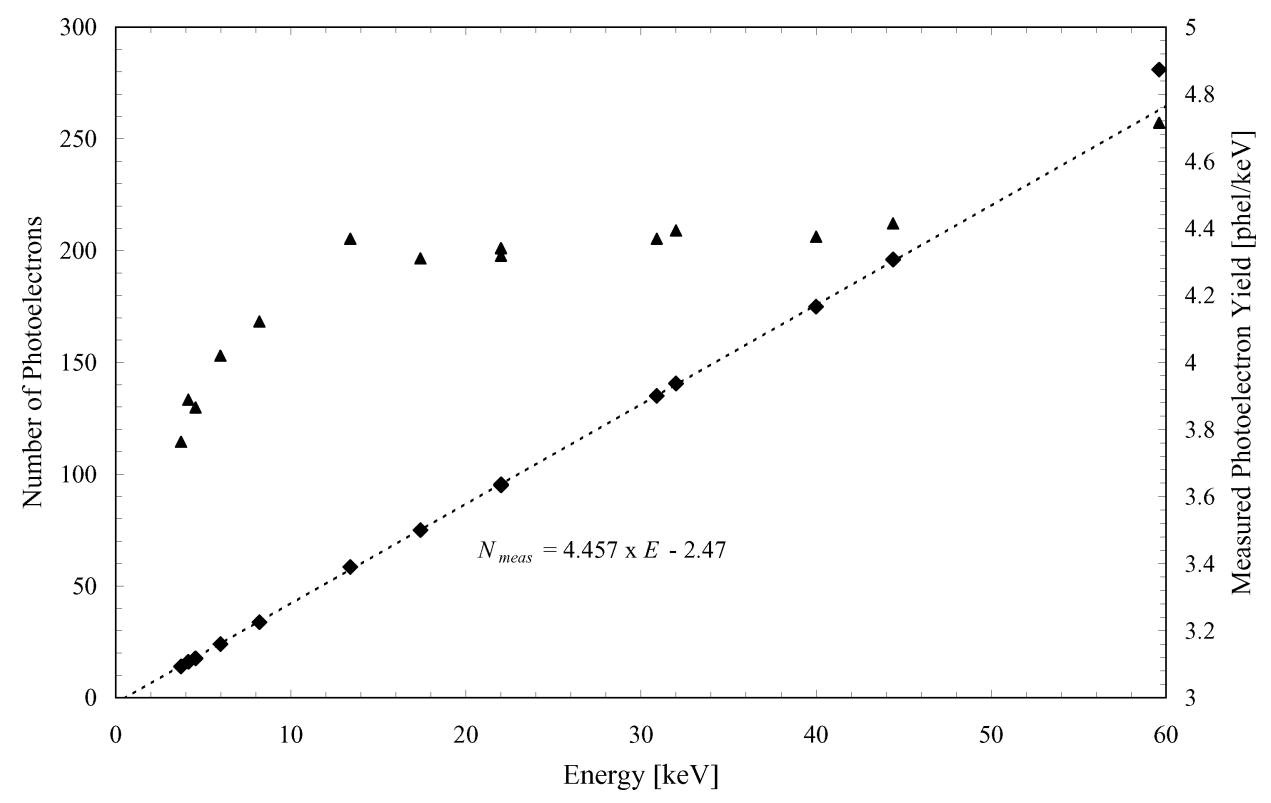

Fig. 5. Measured number of photoelectrons $N_{\text {meas }}$ plotted versus the emitted $\mathrm{K}_{\alpha}$ energies of the indicated radiation sources (left-hand scale). The broken line shows its linear regression fit, which intercepts the $N_{\text {meas }}$ axis at -2.47 phel with a slope of 4.457 phel/keV (the ${ }^{241} \mathrm{Am}$ point is not included in the least square fit procedure). (See Table I.) The right-hand scale shows the measured photoelectron yield in phel/keV, indicating nonproportionality between $N_{\text {meas }}$ and $E$ for low energy values.

ergies between $14 \mathrm{keV}\left({ }^{57} \mathrm{Co}\right)$ and $1275 \mathrm{keV}\left({ }^{22} \mathrm{Na}\right)$. We have visualized almost ten photoelectron peaks and calibrated them in terms of $N_{\text {meas }}$ versus the channels of the pulse-height analyzer, and hence, versus each well-known $\mathrm{K}_{\alpha}$ emission peak energy [example: Fig. 4(b)]. This procedure was repeated for each $\mathrm{X}$-ray or gamma spectrum, and therefore, each total absorption peak could be precisely associated to its photoelectron number $N_{\text {meas }}$ (see also [4]-[6]). In this way, we found $4.46 \mathrm{phel} / \mathrm{keV}$ with the 0.3-mm thick YAP plate inside an HPMT with Be radiation entrance window (Fig. 5). This excellent result is due to the thin YAP-plate, which reduced its self-absorption of scintillation light to a minimum. It is worth noting that it was achieved without any reflective or diffusive cover at the disk's radiation entrance surface (Fig. 1).

Moszynski et al. and Kapusta et al. report low values for energy resolutions, e.g., 5.7\% and 4.38\% at FWHM for $662 \mathrm{keV}$ $\left({ }^{137} \mathrm{Cs}\right)$ ([2, Fig. 4] and [3, Fig. 1], respectively). Our energy resolution (17\%) for ${ }^{241} \mathrm{Am}(59.6 \mathrm{keV})$ in Fig. 4(a) is rather close to that of Moszynski et al. (16.1\%) [2, Figs. 6 and 7]. As the detector is mainly meant to detect X-rays [and its $\mathrm{YAP}(\mathrm{Ce})$ disk is kept thin], an extrapolation to the $662 \mathrm{keV}\left({ }^{137} \mathrm{Cs}\right)$ line would heavily rely on the model chosen.

The difference between the purely statistical contribution and the presently measured resolutions (Fig. 6) merits some remarks. The energy resolution at FWHM for our detector can be approximated as following:

$$
\begin{aligned}
\left(\frac{\Delta E}{E}\right)_{\text {tot }}^{2} & =\left(\frac{\Delta E}{E}\right)_{\text {int } r}^{2}+\left(\frac{\Delta E}{E}\right)_{\text {trans }}^{2} \\
& +\left(\frac{0.3}{N(E)_{\text {meas }}}\right)_{\text {elec }}^{2}+\left(\frac{2.36 \cdot(1+\varepsilon)}{\sqrt{N(E)_{\text {meas }}}}\right)_{\text {stat }}^{2}
\end{aligned}
$$

where the sum in quadrature means the contributions from the intrinsic, transition, electronic, and statistical terms. Generally, the intrinsic term refers to the crystal best possible energy resolution and it can be a constant or a slowly varying quantity [7]. It rises from local variations of: scintillation yield, due to Ce-doping and energy conversion processes, and to light propagation in the crystal, caused by small defaults or color centers. The transition term indicates the worsening in resolution due to the light transfer from the crystal to the photocathode. It involves also the geometrical crystal shape and the diffusive or reflective coatings usually added to the crystal surface. In our case, it is mainly due to: photocathode disuniformity, a very high surface-to-volume ratio of the $\mathrm{YAP}(\mathrm{Ce})$ disk (for example causing many internal light reflections) and to a photocathode-crystal surface ratio smaller than one. The electronic term is present only for low photoelectron numbers with a coefficient of $\sim 0.3$, measured from the FWHM of the single photoelectron response distribution [1]. The factor $\varepsilon$, indicated in the statistical term, is mainly caused by photoelectron backscatterings on the silicon anode surface and, in our case, by variations of the overall efficiency over the photocathode surface and it should be small for HPMTs as compared to photomultipliers [1], [8]. In the following, we shall try to qualitatively understand the contributions of the energy resolution terms to the measured values and to understand their possible origin. A word of caution is indispensable at this point, as all these processes are interrelated (especially in this case). Therefore, to split the energy resolution in different contributions is a rough approximation and has to be taken as such.

Measured energy resolutions are listed in Table I and are plotted in Fig. 6 versus the number of measured photoelectrons $N_{\text {meas }}$. The triangles indicate the results obtained with the Be-YAP HPMT, while the squares indicate the values ob- 


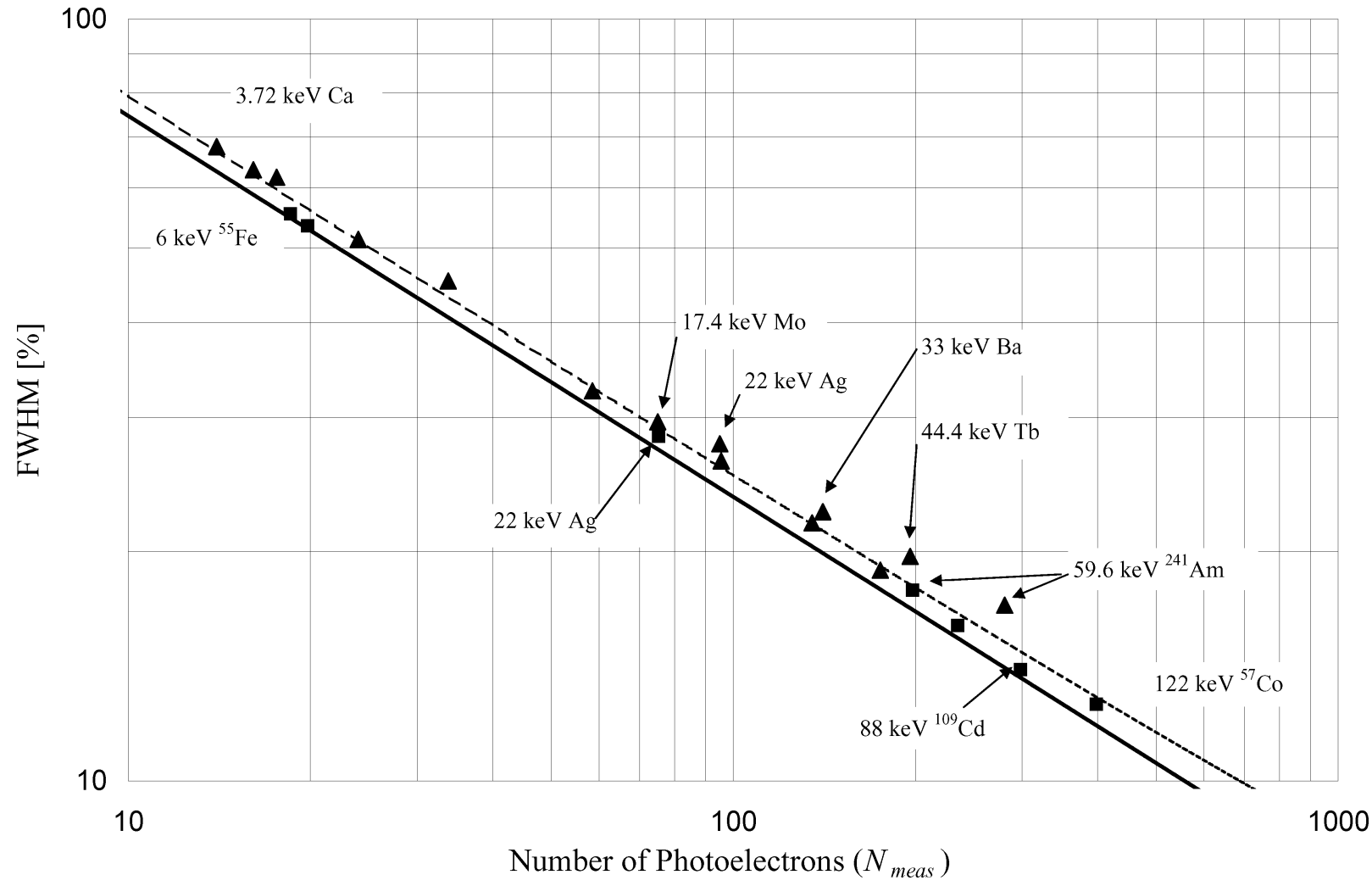

Fig. 6. Measured energy resolutions (FWHM) versus the measured number of photoelectrons $N_{\text {meas }}$, obtained for the $\mathrm{K}_{\alpha}$ energies of the indicated elements (triangles). The solid line shows the resolutions expected from photoelectron statistics only. The broken line is calculated from relation (1), inserting the values $\varepsilon=0.06,(\Delta E / E)_{\text {intr }}=0.03,(\Delta E / E)_{\text {elec }}=0.3 / N_{\text {meas }}$ and considering $(\Delta E / E)_{\text {trans }}=0$. The squares are taken with the "standard" configuration, as described in Section III [5]. They show better resolutions at equivalent photoelectron numbers $N_{\text {meas }}$. (See Table I.)

TABLE I

Values of $N_{\text {meas }}$ and Measured Resolutions at FWhM for Be-YAP(Ce) (See Fig. 1) and "Standard” Configurations [See Insert of Fig. 4(B)]. They are Plotted in Figs. 5 And 6. Pay Attention to the Different Photoelectron Numbers $N_{\text {meas }}$ Associated With the Same Energies for Both Configurations. *X-RAy Lines EXcited by ${ }^{241}$ Am Gammas (See TeXT). **The Disk 1-Mm-Thin Sides Were Also WrapPed

\begin{tabular}{|c|c|c|c|c|c|c|}
\hline \multirow[b]{2}{*}{ Sources } & \multicolumn{3}{|c|}{ Be - YAP configuration } & \multicolumn{3}{|c|}{ "Standard" configuration } \\
\hline & $E[\mathrm{keV}]$ & $N_{\text {meas }}$ & $(\Delta E / E)_{t o t}[\%]$ & $E[\mathrm{keV}]$ & $N_{\text {meas }}$ & $(\Delta E / E)_{t o t}[\%]$ \\
\hline $\mathrm{Ca}$ & 3.72 & 14 & 68 & & & \\
\hline $\mathrm{Sc}$ & 4.14 & 16.1 & 63.4 & & & \\
\hline $\mathrm{Ti}$ & 4.55 & 17.59 & 62 & & & \\
\hline${ }^{55} \mathrm{Fe}(\mathrm{Mn} \mathrm{K})$ & 6.0 & 24 & 51.4 & 6.0 & 18.53 & 55.5 \\
\hline${ }^{57} \mathrm{Co}(\mathrm{Fe} \mathrm{K})$ & & & & 6.5 & 19.8 & 53.54 \\
\hline $\mathrm{Cu}$ & 8.2 & 33.8 & 45.3 & & & \\
\hline $\mathrm{Rb}$ & 13.39 & 58.5 & 32.5 & & & \\
\hline Mo & 17.4 & 75 & 29.6 & & & \\
\hline${ }^{109} \mathrm{Cd}$ & 22 & 95 & 26.3 & 22 & 75.3 & 28.32 \\
\hline $\mathrm{Ag}^{*}$ & 22 & 95.5 & 27.7 & & & \\
\hline${ }^{133} \mathrm{Ba}(\mathrm{Cs} \mathrm{K})$ & 30.9 & 135 & 21.8 & & & \\
\hline $\mathrm{Ba}^{*}$ & 32 & 140.6 & 22.56 & & & \\
\hline${ }^{152} \mathrm{Eu}(\mathrm{Sm} \mathrm{K})$ & 40 & 175 & 18.9 & & & \\
\hline $\mathrm{Tb}^{*}$ & 44.4 & 196 & 19.7 & & & \\
\hline${ }^{241} \mathrm{Am}$ & 59.6 & 281 & 17 & & $198(235)^{* *}$ & $17.8(16)^{* *}$ \\
\hline${ }^{109} \mathrm{Cd}$ & & & & 88 & 298.45 & 14 \\
\hline${ }^{57} \mathrm{Co}$ & & & & 122 & 398 & 12.6 \\
\hline
\end{tabular}


tained in the "standard" configuration (see Section III). The lower straight line follows the pure statistical term with $\varepsilon=0$ (see relation 1). In addition, the upper broken line follows relation (1) with $\varepsilon=0.06,(\Delta E / E)_{\mathrm{intr}}=0.03,{ }^{3}(\Delta E / E)_{\mathrm{elec}}=$ $0.3 / N_{\text {meas }}$ and $(\Delta E / E)_{\text {trans }}=0$ and, in our range of energies, represents much better the measured values (triangles) for the Be-YAP HPMT. ${ }^{4}$ On the contrary, the equivalent energy resolution values, measured with the "standard" configuration (squares), follow much closer the purely statistical contribution line (solid line) and confirm the low $\varepsilon$-values, as expected from [8] for HPMTs. Finally, we also note the $\sim 5 \%$ difference in $N_{\text {meas }}$ values between center and off-center positions for the Be-YAP configuration, as displayed in Fig. 3(a). The comparison between the two configurations confirms that the apparent higher $\varepsilon$ for the Be-YAP configuration is a consequence of the particular shape and size of the YAP-disk $(22 \mathrm{~mm}$ diameter and $0.3 \mathrm{~mm}$ thickness) and of its smaller diameter $(\sim 17 \mathrm{~mm})$ photocathode.

\section{CONCLUSION}

Our first application of mounting a YAP-disk inside an HPMT was successful. The linear relation between photoelectron numbers and energies was: $N_{\text {meas }}=4.457 \times E-2.47$ (Fig. 5), and the energy resolution (FWHM) follows a relation close to $(\Delta E / E)_{\text {tot }} \approx\left((2.36 \cdot 1.06) / \sqrt{N_{\text {meas }}}\right)_{\text {stat }}$. The intrinsic and transition terms are significant only at higher photoelectron numbers; the electronic term becomes visible only at lower photoelectron numbers and the apparent higher $\varepsilon$ for the Be-YAP configuration seems to be a consequence of its specific window geometry. Thus, the previous relation

${ }^{3}$ The intrinsic term of $3 \%$ is a rough estimation based on references [2] and [3], which indicate $3.4 \%$ and $1.3 \%$, respectively.

${ }^{4}$ It is worth noting that, in the Be-YAP configuration, the resolutions of the ${ }^{241} \mathrm{Am} \gamma$-line and of the $\mathrm{Ag}, \mathrm{Ba}$ and $\mathrm{Tb} \mathrm{X}$-ray lines are worse than expected. This is due to two high activity ${ }^{241} \mathrm{Am}$ sources of $36 \mathrm{MBq}$ (point source) and $350 \mathrm{MBq}$ (ring source to activate the K-lines). In fact, the ${ }^{109} \mathrm{Cd} 22 \mathrm{keV}$ line, the ${ }^{133} \mathrm{Ba} 30.9 \mathrm{keV}$ line and the ${ }^{152} \mathrm{Eu} 40 \mathrm{keV}$ line from the respective sources, which have energies close to the aforementioned X-ray lines, feature lower resolution values at the same or roughly the same number of measured photoelectrons. translates in energy units as $(\Delta E / E)_{\text {tot }} \approx(0.56 / \sqrt{E[k e V]})$ for $5 \mathrm{keV} \leq E \leq 60 \mathrm{keV}$.

The next step consists in mounting a thin YAP-plate inside an ISPA-tube and sealing it again with a Be-window. This should allow for spatial resolutions of the order of $\sim 0.3 / \sqrt{N_{\text {meas }}} \mathrm{mm}$ for single-event detection and $\sim 0.3 \mathrm{~mm}$ for $\mathrm{X}$-ray bursts. Thin $\mathrm{YAP}(\mathrm{Ce})$ disks of $0.1 \mathrm{~mm}$ have also been produced and will be eventually employed to further improve spatial resolution.

\section{ACKNOWLEDGMENT}

The authors wish to thank H.J. Hilke, W. Klempt, and O. Ullaland for the careful and critical reading of the manuscript. All the measurements and the technical development of the Be-YAP HPMT were performed with the support of the TA2 group at CERN. They particularly acknowledge the help of F. Cindolo and D. Piedigrossi.

\section{REFERENCES}

[1] C. D'Ambrosio and H. Leutz, "Hybrid photon detectors," Nucl. Instrum. Methods Phys. Res. A, vol. A501, pp. 463-498, 2003.

[2] M. Moszynski, M. Kapusta, D. Wolski, W. Klamra, and B. Cederwall, "Properties of the YAP:Ce scintillator," Nucl. Instrum. Methods Phys. Res. A, vol. A404, pp. 157-165, 1998.

[3] M. Kapusta, M. Balcerzyk, M. Moszynski, and J. Pawelke, "A highenergy resolution observed from a YAP:Ce scintillator," Nucl. Instrum. Methods Phys. Res. A, vol. A421, pp. 610-613, 1999.

[4] C. D'Ambrosio, F. De Notaristefani, H. Leutz, D. Puertolas, and E. Rosso, "X-ray detection with a scintillating YAP-window hybrid photomultiplier tube," IEEE Trans. Nucl. Sci., vol. 47, no. 1, pp. 6-12, Feb. 2000

[5] C. D'Ambrosio, C. Ercoli, S. Jaaskelainen, G. Lecoeur, H. Leutz, R. Loos, D. Piedigrossi, D. Puertolas, E. Rosso, and R. Schomaker, "A HMPT based set-up to characterize scintillating crystals," Nucl. Instrum. Methods Phys. Res. A, vol. A434, pp. 387-398, 1999.

[6] F. Cindolo, C. D'Ambrosio, F. De Notaristefani, H. Leutz, D. Piedigrossi, D. Puertolas, and E. Rosso, "ISPA tubes with scintillating YAP:Ce windows:X- and $\gamma$-ray imaging," IEEE Trans Nucl. Sci., vol. 50, no. 1, pp. 126-132, Feb. 2003.

[7] M. Moszynski, J. Kalipska, M. Balcerzyk, M. Kapusta, W. Mengesha, and J. D. Valentine, "Intrinsic energy resolution of NAI(Tl)," Nucl. Instrum. Methods Phys. Res. A, vol. A484, pp. 259-269, 2002.

[8] C. D'Ambrosio and H. Leutz, "Photoelectron backscattering from silicon anodes of hybrid photodetector tubes," IEEE Trans Nucl. Sci., vol. 47, no. 4, pp. 1685-1690, Aug. 2000. 